Series A

I. MATHEMATICA

411

\title{
THE COEFFICIENTS OF QUASICONFORMALITY OF ELLIPSOIDS
}

BY

G. D. ANDERSON

H E L S N K I 1967

S U O M A L A I E N T I D D A K A T E I A 
Communicated 10 February 1967 by Oldi Lehto and K. I. Virtanen 


\section{THE COEFFICIENTS OF QUASICONFORMALITY OF ELLIPSOIDS ${ }^{1}$ )}

\section{Introduction}

The problem of extremal plane quasiconformal mappings was first treated by $\mathrm{H}$. Grötzsch [12], who proved that the extremal quasiconformal mapping of one rectangle onto another with vertices corresponding in the same order is the affine mapping. As observed by Ahlfors [1], this actually shows that the extremal quasiconformal mapping of one quadrilateral onto another is the composition of a conformal mapping, an affine mapping, and a second conformal mapping. Moreover, the work of Teichmüller [17] shows that there is a large class of extremal quasiconformal mappings that are given locally by such a sequence of mappings.

Next, suppose that $Z$ and $Z^{\prime}$ are finite right circular cylinders in $R^{3}$ with bases of equal area and with lengths $L$ and $L^{\prime}$, respectively. It is easy to show by means of an extremal lengths argument that the natural affine mapping of $Z$ onto $Z^{\prime}$ is extremal among all quasiconformal maps of $Z$ onto $Z^{\prime}$ that preserve the bases. Moreover, from this and Theorem 11.2 of [10] it follows that the affine map is extremal among all quasiconformal mappings of $Z$ onto $Z^{\prime}$ when $2^{-\frac{1}{2}} \leq L^{\prime} \mid L \leq 2^{\frac{1}{4}}$.

For such reasons it is then natural to ask if in 3 -space the affine mapping is extremal in the class of all quasiconformal homeomorphisms of an ellipsoid onto the unit ball. In the present paper we disprove this conjecture in several cases by constructing mappings with lower dilatations than those of the affine mapping. In several instances our work also gives the correct order for the coefficients of quasiconformality. For the prolate ellipsoid these coefficients are bounded, while in another case the dilatation of the affine map has the same order as that of an extremal mapping.

Before attacking these problems, we introduce in the next section some basic terminology and results, most of which are contained in [10].

1) This research was supported in part by the National Science Foundation, Grant 18913, and by a Fellowship from the Institute of Science and Technology in 1965. The work is part of a Ph.D. thesis written under the direction of Professor F. W. Gehring at the University of Michigan. 
A. I. 411

\section{Quasiconformal mappings}

Suppose that $f$ is a diffeomorphism of a 3 -space domain $\Omega$ onto $\Omega^{\prime}$. Then $f$ is locally affine - if $P \in \Omega$ the differential mapping $d f(P)$ takes the unit ball onto an ellipsoid with semiaxes of lengths, say, $a \geq b \geq c$. Then each of the dilatation functions

$$
H_{I}(P, f)=\frac{a b}{c^{2}}, H_{o}(P, f)=\frac{a^{2}}{b c}
$$

is greater than or equal to unity, with equality at a point $P$ if and only if $f$ is conformal there. Moreover,

$$
H_{I}(P, f) \leq H_{o}(P, f)^{2}, H_{o}(P, f) \leq H_{I}(P, f)^{2}
$$

for each $P \in \Omega$.

Next, the functionals

$$
K_{I}(f)=\sup _{P \in \Omega} H_{I}(P, f), K_{o}(f)=\sup _{P \in \Omega} H_{o}(P, f)
$$

are called the inner and outer dilatations ${ }^{2}$ ) of $f$, respectively; each is a natural measure of how far $f$ is from being a Möbius transformation of $\Omega$. Then

$$
K_{I}(f) \leq K_{o}(f)^{2}, K_{o}(f) \leq K_{I}(f)^{2},
$$

so that these dilatations are both infinite or finite. If both are bounded by $K, 1 \leq K<\infty$, then $f$ is called a $K$-quasiconformal mapping. A quasiconformal mapping is one which is $K$-quasiconformal for some $K$.

The above definitions may be generalized, by means of extremal lengths, to include an arbitrary homeomorphism $f$ of $\Omega$ onto $\Omega^{\prime}$. If $\Gamma$ is a family of arcs in $R^{3}$, we let $F(\Gamma)$ denote the family of density functions $\varrho$ that are nonnegative and Borel-measurable in $R^{3}$ and for which

$$
\int_{\gamma} \varrho d s \geq 1
$$

for each $\gamma \in \Gamma$. If $\gamma$ is not locally rectifiable, the integral is taken with respect to linear measure [16]. Then following Väisälä [18] we define the modulus $M(\Gamma)$ of the arc family $\Gamma$ as

$$
M(\Gamma)=\inf _{\varrho} \int_{R^{3}} \varrho^{3} d \omega,
$$

$\left.{ }^{2}\right)$ Sometimes the inner and outer dilatations are defined as the square root of these expressions. 
where the infimum is taken over all $\varrho \in F(\Gamma)$ (See also [6] and [10]). Given an arbitrary homeomorphism $f$ of $\Omega$ onto $\Omega^{\prime}$, the inner and outer dilatations of $f$ are defined by

$$
K_{I}(f)=\sup _{I^{\prime}} \frac{M(f(\Gamma))}{M(\Gamma)}, K_{o}(f)=\sup _{\Gamma} \frac{M(\Gamma)}{M(f(\Gamma))},
$$

where the suprema are taken over all families $\Gamma$ in $\Omega$ for which $M(\Gamma)$ and $M(f(\Gamma))$ are not both 0 or $\infty^{2}$ ).

It is easily verified that these definitions agree with (3) when $f$ is a diffeomorphism. Moreover, it follows from Lemma 1.2 of [10] and Theorem 5 of [9] that inequalities (4) hold also for definitions (6), hence that $K_{I}(f)$ and $K_{o}(f)$ are both infinite or finite. In the latter case, $f$ is said to be quasiconformal; $f$ is $K$-quasiconformal if

$$
K(f)=\max \left(K_{I}(f), K_{o}(f)\right) \leq K .
$$

Finally, given a domain $\Omega$ homeomorphic to the unit ball $B^{3}$, let

$$
K_{I}(\Omega)=\inf _{f} K_{I}(f), K_{o}(\Omega)=\inf _{f} K_{o}(f)
$$

where the infima are taken over all homeomorphisms $f$ of $\Omega$ onto $B^{3}$. If $\Omega$ is not homeomorphic to $B^{3}$, set $K_{I}(\Omega)=K_{o}(\Omega)=\infty$. The numbers $K_{I}(\Omega)$ and $K_{o}(\Omega)$ are called the inner and outer coefficients of quasiconformality of $\Omega$, respectively. From (4) we have

$$
K_{I}(\Omega) \leq K_{o}(\Omega)^{2}, K_{o}(\Omega) \leq K_{I}(\Omega)^{2},
$$

so that these coefficients are both infinite or finite. In the latter case, $\Omega$ is called a quasiconformal ball. By (8) clearly $K_{I}(\Omega) \geq 1$ and $K_{0}(\Omega) \geq 1$; by Liouville's Theorem (Theorem 15 of [9]) one, and hence both, of these coefficients is 1 if and only if $\Omega$ is a ball or half space.

\section{The prolate ellipsoid}

We consider first the prolate ellipsoid. We prove

Theorem 1. Let $E_{p}$ be any prolate ellipsoid. Then the affine mapping of $E_{p}$ onto the unit ball is not extremal for either the inner or the outer coefficient. Both coefficients of a prolate ellipsoid are bounded.

Proof. According to [13, p. 177] or [14, pp. 295, 296], the plane mapping

$$
y_{1}+i y_{2}=f\left(x_{1}+i x_{2}\right)=k^{\frac{1}{2}} \text { sn }\left(\frac{2 K}{\pi} \zeta, k\right)
$$


maps the ellipse $x_{1}^{2} / a^{2}+x_{2}^{2} / b^{2}<1$ conformally onto the unit disk, where $x_{1}+i x_{2}=\sin \zeta$ and $\zeta=\alpha+i \beta$. Here $K$ and $K^{\prime}$ are the elliptic integrals defined by

$$
\begin{aligned}
K & =K(k)=\int_{0}^{1}\left[\left(1-x^{2}\right)\left(1-k^{2} x^{2}\right)\right]^{-\frac{1}{2}} d x, \\
K^{\prime} & =K\left(k^{\prime}\right), k^{\prime}=\left(1-k^{2}\right)^{\frac{1}{2}},
\end{aligned}
$$

sn denotes the Jacobian elliptic sine function, and

$$
a=\cosh t, b=\sinh t, t=\frac{\pi K^{\prime}}{4 K} .
$$

If the $x_{1}+i x_{2}$ and $y_{1}+i y_{2}$ planes are rotated about the real axes, there results a diffeomorphism $y=f_{p}(x)$ of the prolate ellipsoid $E_{p}$ : $x_{1}^{2} / a^{2}+x_{2}^{2} / b^{2}+x_{3}^{2} / b^{2}<1$ onto the unit ball. That is, we let

$$
y_{1}+i s=f\left(x_{1}+i r\right), \varphi=\theta \text {, }
$$

where $(r, \theta)$ and $(s, \varphi)$ are polar coordinates in the $x_{2} x_{3}$ and $y_{2} y_{3}$ planes, respectively.

In determining the dilatations of $f_{p}$ it is convenient to make use of the hyperbolic density [15]. Suppose $\Omega$ is a simply-connected plane domain with more than one boundary point, in the $\zeta$ plane, and let $\omega(\zeta)$ be a conformal mapping of $\Omega$ onto the upper half plane $\operatorname{Im} \omega>0$. Then the hyperbolic density for $\Omega$ is defined to be

$$
\varrho(\zeta)=\varrho(\zeta, \Omega)=\frac{\left|\frac{d \omega}{d \zeta}\right|}{2 \operatorname{Im} \omega} .
$$

Hence the hyperbolic density $\varrho_{1}$ of the upper half plane $\Omega_{1}: x_{2}>0$, $x_{3}=0$ is

$$
\varrho_{1}=\varrho\left(x_{1}+i x_{2}, \Omega_{1}\right)=\frac{1}{2 x_{2}} .
$$

Next, by virtue of the reflection principle, the plane mapping $f\left(x_{1}+i x_{2}\right)$ carries the semi-ellipse $\Omega_{2}: x_{1}^{2} / \cosh ^{2} 2 t+x_{2}^{2} / \sinh ^{2} 2 t<1, x_{2}>0, x_{3}=0$ conformally onto the upper half plane $y_{2}>0, y_{3}=0$. Hence the hyperbolic density of $\Omega_{2}$ is

$$
\varrho_{2}=\varrho\left(x_{1}+i x_{2}, \Omega_{2}\right)=\frac{\left|f^{\prime}\left(x_{1}+i x_{2}\right)\right|}{2 y_{2}} .
$$


From (12) and (13) and the fact that the hyperbolic density $\varrho(\zeta, \Omega)$ is a monotonic decreasing function of domain we arrive at the following form of the Schwarz Lemma: For all points in $\Omega_{2}$,

$$
\frac{\varrho_{2}}{\varrho_{1}}=\frac{x_{2}}{y_{2}}\left|f^{\prime}\right| \geq 1 \text {. }
$$

It is now easy to see, using (3) and (14), that

$$
K_{I}\left(f_{p}\right)=\sup \left(\frac{x_{2}}{y_{2}}\left|f^{\prime}\right|\right)^{2}, K_{o}\left(f_{p}\right)=\sup \left(\frac{x_{2}}{y_{2}}\left|f^{\prime}\right|\right),
$$

where the suprema are taken over all points $\left(x_{1}, x_{2}, 0\right)$ in the ellipse $x_{1}^{2} / a^{2}+x_{2}^{2} / b^{2}<1, x_{3}=0$, with $a$ and $b$ as in (10).

Next we use the fact that the hyperbolic density $\varrho$ satisfies the differential equation $\Delta \log \varrho=4 \varrho^{2}$, where $\Delta$ is the Laplacian operator. From this fact and the inequality (14) it follows that

$$
\Delta \log \frac{\varrho_{2}}{\varrho_{1}}=4\left(\varrho_{2}^{2}-\varrho_{1}^{2}\right) \geq 0
$$

in $\Omega_{2}$ and hence, by symmetry and continuity, throughout the ellipse $x_{1}^{2} / a^{2}+$ $x_{2}^{2} / b^{2}<1, x_{3}=0$. From (16) we conclude that log $\left(\varrho_{2} / \varrho_{1}\right)$ is subharmonic in this ellipse as a function of $x_{1}$ and $x_{2}$. Consequently the suprema in (15) must occur on the boundary. In fact, by symmetry, it is clear that the suprema will be achieved on the arc $\gamma: x_{1}^{2} / a^{2}+x_{2}^{2} / b^{2}=1, x_{2} \geq 0, x_{3}=0$.

Since $\beta=t=\pi K^{\prime} / 4 K$ on $\gamma$, where $x_{1}+i x_{2}=\sin (\alpha+i \beta)$,

$$
f\left(x_{1}+i x_{2}\right)=k^{\frac{1}{2}} \operatorname{sn}\left(\frac{2 K}{\pi} \alpha+i \frac{K^{\prime}}{2}, k\right)
$$

there. Then use of the addition theorem for sn $([4$, p. 38], $[5, \# 125.01])$, the special values $\operatorname{sn}\left(K^{\prime} / 2, k^{\prime}\right)=(1+k)^{-\frac{1}{2}}, \quad \operatorname{cn}\left(K^{\prime} / 2, k^{\prime}\right)=[k /(1+k)]^{\frac{1}{2}}$, $\operatorname{dn}\left(K^{\prime} / 2, k^{\prime}\right)=k^{\frac{1}{2}}\left([\tilde{5}, \# 122.10], \quad\left[11\right.\right.$, p. 120]), and the identity $k^{2} \operatorname{sn}^{2} u$ $=1-\operatorname{dn}^{2} u([4$, p. 9], $[5, \# 121.00])$ replaces $(17)$ by

$$
f\left(x_{1}+i x_{2}\right)=\frac{(1+k) \cdot s+i c d}{1+k s^{2}}
$$

on $\gamma$. Here we have employed the notation

$$
s=\operatorname{sn}\left(\frac{2 K}{\pi} \alpha, k\right), c=\operatorname{cn}\left(\frac{2 K}{\pi} \alpha, k\right), d=\operatorname{dn}\left(\frac{2 K}{\pi} \alpha, k\right) .
$$

Finally, employing the Gauss Transformation ([4, pp. 72, 73], [5, \#164.01 $-.02])$ we may reduce (19) to

$$
f\left(x_{1}+i x_{2}\right)=\operatorname{sn}\left(\frac{2 \Lambda}{\pi} \alpha, \lambda\right)+i \operatorname{cn}\left(\frac{2 \Lambda}{\pi} \alpha, \lambda\right)
$$


on $\gamma$, where

$$
\begin{aligned}
& \lambda=\frac{2 k^{\frac{1}{2}}}{1+k}, \lambda^{\prime}=\frac{1-k}{1+k}, \\
& \Lambda=K(\lambda)=(1+k) K, \Lambda^{\prime}=K\left(\lambda^{\prime}\right)=(1+k) \frac{K^{\prime}}{2} .
\end{aligned}
$$

Then differentiation of (20) with respect to $x_{1}+i x_{2}$ and use of the relations $(\operatorname{sn} u)^{\prime}=\operatorname{cn} u \operatorname{dn} u,(\operatorname{cn} u)^{\prime}=-\operatorname{sn} u \operatorname{dn} u$ and the identity $\operatorname{sn}^{2} u$ $+\mathrm{cn}^{2} u=1$ yields

$$
\left|f^{\prime}\right|=\frac{2 \Lambda}{\pi} \frac{\operatorname{dn}\left(\frac{2 \Lambda}{\pi} \alpha, \lambda\right)}{\left[\cos ^{2} \alpha+\sinh ^{2} t\right]^{\frac{1}{2}}} .
$$

But on the $\operatorname{arc} \gamma, x_{2}=\operatorname{Im} \sin (\alpha+i t)=\cos \alpha \sinh t$, and with (20) and (22) we achieve

$$
\frac{x_{2}}{y_{2}}\left|f^{\prime}\right|=\frac{2 \Lambda}{\pi} \frac{\operatorname{dn}\left(\frac{2 \Lambda}{\pi} \alpha, \lambda\right)}{\operatorname{cn}\left(\frac{2 \Lambda}{\pi} \alpha, \lambda\right)} \frac{\cos \alpha \sinh t}{\left[\cos ^{2} \alpha+\sinh ^{2} t\right]^{\frac{1}{2}}} .
$$

The value of $(23)$ is easily seen to be $\frac{2 \Lambda}{\pi} \tanh t$ when $\alpha=0$. But since

$$
t=\frac{\pi K^{\prime}}{4 K}=\frac{\pi \Lambda^{\prime}}{2 \Lambda}
$$

by (10) and (21), it follows that the suprema in (15) are achieved for $\alpha=0$ if and only if the following holds:

Lemma 1. For each $\lambda, 0 \leq \lambda<1$, and all $\alpha,-\pi / 2 \leq x \leq \pi / 2$,

$$
\frac{\operatorname{dn}^{2}\left(\frac{2 \Lambda}{\pi} \alpha, \lambda\right)\left(\cos ^{2} \alpha\right)\left(\cosh ^{2} \frac{\pi \Lambda^{\prime}}{2 \Lambda}\right)}{\operatorname{cn}^{2}\left(\frac{2 \Lambda}{\pi} \alpha, \lambda\right)} \leq 1 .
$$

A proof of this lemma is given in [3]. Thus (15) becomes

$$
K_{I}\left(f_{p}\right)=\left(\frac{2 \Lambda}{\pi} \tanh \frac{\pi \Lambda^{\prime}}{2 \Lambda}\right)^{2}, K_{o}\left(f_{p}\right)=\frac{2 \Lambda}{\pi} \tanh \frac{\pi \Lambda^{\prime}}{2 \Lambda} .
$$


That these dilatations, and hence the coefficients of $E_{p}$, are bounded is a consequence of the limit

$$
\lim _{\lambda \rightarrow 1} \frac{2 \Lambda}{\pi} \tanh \frac{\pi \Lambda^{\prime}}{2 \Lambda}=\left[\lim _{\lambda \rightarrow 1} \Lambda^{\prime}\right]\left[\lim _{\lambda \rightarrow 1} \frac{\tanh \frac{\pi \Lambda^{\prime}}{2 \Lambda}}{\frac{\pi \Lambda^{\prime}}{2 \Lambda}}\right]=\frac{\pi}{2}
$$

(See also [3]).

We wish to compare the dilatations (25) with those of the natural affine mapping $g_{p}(x)=\left(x_{1} / a, x_{2} / b, x_{3} / b\right)$ of $E_{p}$ onto the unit ball. Since $g_{p}$ is a diffeomorphism it is easy to see that

$$
\begin{aligned}
& K_{I}\left(g_{p}\right)=\left(\frac{a}{b}\right)^{2}=\left(\operatorname{coth} \frac{\pi \Lambda^{\prime}}{2 \Lambda}\right)^{2}, \\
& K_{O}\left(g_{p}\right)=\frac{a}{b}=\operatorname{coth} \frac{\pi \Lambda^{\prime}}{2 \Lambda} .
\end{aligned}
$$

But in [3] we have proved

Lemma 2. For $0 \leq \lambda<1$,

$$
\frac{1}{2}\left(1+\frac{2 \Lambda}{\pi}\right) \tanh \frac{\pi \Lambda^{\prime}}{2 \Lambda} \leq 1,
$$

with strict inequality if $0<\lambda<1$.

But since trivially $\left(\frac{2 \Lambda}{\pi}\right)^{\frac{1}{2}} \leq \frac{1}{2}\left(1+\frac{2 \Lambda}{\pi}\right)$ it follows from this lemma that $K_{I}\left(f_{p}\right)<K_{I}\left(g_{p}\right)$ and $K_{o}\left(f_{p}\right)<K_{o}\left(g_{p}\right)$ when $0<\lambda<1$, so that the affine mapping is not extremal for either coefficient.

\section{The outer coefficient of the oblate ellipsoid}

We turn our attention now to the oblate ellipsoid. We begin by proving

Theorem 2. Let $E_{o}$ be any oblate ellipsoid. Then the affine mapping of $E_{o}$ onto the unit ball is not extremal for the outer coefficient.

Proof. The proof we present is based on a general mapping introduced by Gehring and Väisälä [10]. We define a radial mapping $y=h(x)$ from $E_{o}: x_{1}^{2} / a^{2}+x_{2}^{2} / b^{2}+x_{3}^{2} / a^{2}<1, a>b$ to the unit ball as follows: If $P \in E_{o}, P \neq 0$, then $P$ has a unique representation of the form $P=r Q$, where $Q \in \partial E_{o}$ and $0<r<1$. For each such $P \in E_{o}$ define

$$
h(P)=r^{p} h(Q), h(Q)=\frac{Q}{|Q|},
$$

where $p$ is some fixed positive number, and let $h(0)=0$. 
Next, for each $Q \in \partial E_{o}$ let $\psi(Q)$ denote the acute angle which the tangent plane at $Q$ makes with the ray from 0 to $Q$, and let $\psi=\min$ $\psi(Q)$. An elementary geometric argument shows that $\psi=\arcsin 2 a b /$ $\left(a^{2}+b^{2}\right)$. Now take the exponent $p$ in (27) to be

$$
p=\sin \psi=\frac{2 a b}{a^{2}+b^{2}} .
$$

Then by Theorem 5.1 of [10] it follows that

$$
K_{o}(h) \leq 2^{\frac{1}{2}} \cot \frac{\psi}{2} \cos \frac{\psi}{2}=\frac{a}{b}\left(\frac{2 a^{2}}{a^{2}+b^{2}}\right)^{\frac{1}{2}} .
$$

For the affine mapping $g_{o}$ of $E_{o}$ onto the unit ball we have

$$
K_{o}\left(g_{o}\right)=\left(\frac{a}{b}\right)^{2} \text {. }
$$

But comparison of (29) and (30) shows that $K_{o}\left(g_{o}\right)>K_{o}(h)$ because $a>b$. We conclude that the affine mapping is not extremal for the outer coefficient.

\section{The inner coefficient of the oblate ellipsoid}

In Theorem 1 we showed that for the prolate ellipsoid $E_{p}: x_{1}^{2} / a^{2}+x_{2}^{2} / b^{2}$ $+x_{3}^{2} / b^{2}<1$ the affine mapping $g_{p}$ has the dilatations $K_{I}\left(g_{p}\right)=(a / b)^{2}$ and $K_{o}\left(g_{p}\right)=a / b$, while the coefficients of $E_{p}$ are of the order $O(1)$ as $a / b$ tends to $\infty$. Thus for large $a / b$ the affine mapping is very far from being extremal.

The situation is similar for the outer coefficient of the oblate ellipsoid $E_{o}: x_{1}^{2} / a^{2}+x_{2}^{2} / b^{2}+x_{3}^{2} / a^{2}<1, a>b$. For the affine mapping $g_{o}$ has outer dilatation $K_{o}\left(g_{o}\right)=(a / b)^{2}$, while in Theorem 2 there was given a mapping $h$ of $E_{o}$ onto the unit ball such that

$$
K_{o}(h) \leq \frac{a}{b}\left(\frac{2 a^{2}}{a^{2}+b^{2}}\right)^{\frac{1}{2}}=O\left(\frac{a}{b}\right)
$$

as $a / b$ tends to $\infty$.

The problem of determining whether the affine mapping of an oblate ellipsoid is extremal for the inner coefficient appears to be more difficult. For in this case Theorem 3 below shows that the inner dilatation of the affine map has the same order as $K_{I}\left(E_{o}\right)$ as $a / b$ tends to $\infty$. 
Theorem 3. Let $E_{o}$ be the oblate ellipsoid $x_{1}^{2} / a^{2}+x_{2}^{2} / b^{2}+x_{3}^{2} / a^{2}<1$, $a>b$. Then $K_{I}\left(E_{o}\right) /(a / b)$ is monotonically decreasing as a/b tends to $\infty$, and

$$
\lim _{a / b \rightarrow \infty} \frac{K_{I}\left(E_{o}\right)}{(a / b)}=A
$$

where $0.22<A \leq 1$.

Proof. Since the affine mapping $g_{o}$ of $E_{o}$ onto the unit ball has inner dilatation $K_{I}\left(g_{o}\right)=a / b$, we see immediately that $K_{I}\left(E_{o}\right) /(a / b) \leq 1$ for all values of $a / b$.

For convenience we now assume, as we obviously may, that $b=1$. To prove the monotoneity, let $f_{1}$ be any quasiconformal mapping of $E_{o}=E_{o}\left(a_{1}\right)$ onto the unit ball and let

$$
x^{\prime}=g(x)=\left(a_{1} x_{1} / a_{2}, x_{2}, a_{1} x_{3} / a_{2}\right)
$$

be the natural affine mapping of $E_{o}\left(a_{2}\right)$ onto $E_{o}\left(a_{1}\right)$, where $a_{2}>a_{1}$. Then $y=f_{2}(x)$ is a quasiconformal mapping of $E_{o}\left(a_{2}\right)$ onto the unit ball, where $f_{2}=f_{1} \circ g$. It is easy to see that $K_{I}(g)=a_{2} / a_{1}$, and by $(6)$ there results

$$
K_{I}\left(f_{2}\right) \leq K_{I}\left(f_{1}\right) K_{I}(g)=\frac{a_{2}}{a_{1}} K_{I}\left(f_{1}\right) .
$$

Taking the infimum first over all $f_{2}$ and then over all $f_{1}$ in (32) we achieve

$$
K_{I}\left(E_{o}\left(a_{2}\right)\right) \leq \frac{a_{2}}{a_{1}} K_{I}\left(E_{o}\left(a_{1}\right)\right),
$$

from which the asserted monotoneity follows.

We now obtain the lower bound in the theorem. Our method is similar to an unpublished argument of Gehring and Väisälä. Let $f$ be any quasiconformal mapping of $E_{o}$ onto the unit ball. Let $E_{i}$ denote the arc of the circle $x_{1}^{2}+x_{3}^{2}=a^{2}, x_{2}=0$ that is contained in the closed $i$-th quadrant of $x_{2}=0, i=1,2,3,4$, and let $\Gamma_{1}$ and $\Gamma_{2}$ be the families of arcs that join $E_{1}$ to $E_{3}$ and $E_{2}$ to $E_{4}$, respectively, in $E_{o}$.

Now let $H$ be the portion of $E_{o}$ that is included between the two planes $x_{1}+x_{3}= \pm a$, and let $\varrho$ be the density function which is $2^{-\frac{1}{2}} a^{-1}$ in $H$ and 0 elsewhere. Then $\varrho \in F\left(\Gamma_{1}\right)$. Moreover, an elementary integration shows that

$$
m(H)=\frac{5 \sqrt{2}}{6} \pi a^{2}
$$

Thus

$$
M\left(\Gamma_{1}\right)=M\left(\Gamma_{2}\right) \leq \int_{H} \varrho^{3} d \omega=\frac{m(H)}{2 \sqrt{2} a^{3}}=\frac{5 \pi}{12 a}
$$


Next, by Corollary 3.3 of [10] we have either

$$
M\left(f\left(\Gamma_{1}\right)\right) \geq v(1) \quad \text { or } \quad M\left(f\left(\Gamma_{2}\right)\right) \geq v(1),
$$

where for $r>O, v(r)$ denotes the modulus of the family of arcs which join the segment $-1 \leq x_{1} \leq 0, x_{2}=x_{3}=0$ to the ray $r \leq x_{1} \leq \infty$, $x_{2}=x_{3}=0$ in the half space $x_{2}>0$. By a simple estimate based on Theorem 8 of [2] it is easy to show that

$$
v(1) \geq \frac{2 \pi}{(4.6)^{2}} .
$$

Hence by (33), (34), (35), and (6) we have

$$
K_{I}(f) \geq \max _{i=1,2} \frac{M\left(f\left(\Gamma_{i}\right)\right)}{M\left(\Gamma_{i}\right)}>0.22 a,
$$

so that $K_{I}\left(E_{o}\right)=\inf K_{I}(f)>0.22 a$.

Remark. Let $\stackrel{f}{\Gamma}$ denote the family of curves in $E_{o}$ that join the axis $-1 \leq x_{2} \leq 1, x_{1}=x_{3}=0$ to the circle $x_{1}^{2}+x_{3}^{2}=a^{2}, x_{2}=0$, and let $f$ be any quasiconformal mapping of $E_{o}$ onto the unit ball. In (3.14) of [10] Gehring and Väisälä conjectured that $M(f(\Gamma)) \geq(\pi / q)^{2}$, where $q=2^{\frac{1}{2}} K\left(\left(\frac{1}{2}\right)^{\frac{1}{2}}\right)=2.62 \ldots$ If this conjecture were true, then an argument similar to that given above shows that the lower bound for $A$ in the theorem could be improved to $A>0.39$.

\section{The general ellipsoid}

Finally we consider a general ellipsoid, that is, an ellipsoid which is not necessarily an ellipsoid of revolution. We prove

Theorem 4. Let $E$ be any ellipsoid. Then the affine mapping of $E$ onto the unit ball is not extremal for the outer coefficient.

Proof. By Theorems 1 and 2 we may assume that $E$ is neither prolate not oblate. Thus let $1<b<a<\infty$, and let $E$ denote the ellipsoid $x_{1}^{2} / a^{2}+x_{2}^{2}+x_{3}^{2} / b^{2}<1$. The affine mapping

$$
y=g(x)=\left(x_{1} / a, x_{2}, x_{3} / b\right)
$$

of $E$ onto the unit ball may be regarded as the composition of two affine mappings - an affine mapping

$$
x^{\prime}=g_{1}(x)=\left(b x_{1} / a, x_{2}, x_{3}\right)
$$

of $E$ onto the oblate ellipsoid $E_{o}: x_{1}^{\prime 2} / b^{2}+x_{2}^{\prime 2}+x_{3}^{\prime 2} / b^{2}<1$, followed by a second affine mapping 


$$
y=g_{2}\left(x^{\prime}\right)=\left(x_{1}^{\prime} / b, x_{2}^{\prime}, x_{3}^{\prime} / b\right)
$$

of $E_{o}$ onto the unit ball.

It is easy to see that $K_{o}(g)=a b, K_{o}\left(g_{1}\right)=a / b$, and $K_{0}\left(g_{2}\right)=b^{2}$. Consequently

$$
K_{o}(g)=K_{o}\left(g_{1}\right) K_{o}\left(g_{2}\right)
$$

Now let $f_{2}$ be the quasiconformal mapping of the oblate ellipsoid $E_{o}$ given in (27). Then the composition of $g_{1}$ with $f_{2}$ is a new quasiconformal mapping $h$ of $E$ onto the unit ball. Hence by (6), Theorem 2, and (36), we have

$$
K_{o}(h) \leq K_{o}\left(g_{1}\right) K_{o}\left(f_{2}\right)<K_{o}\left(g_{1}\right) K_{o}\left(g_{2}\right)=K_{o}(g) .
$$

This completes the proof of Theorem 4.

We return in conclusion to the notation $x_{1}^{2} / a^{2}+x_{2}^{2} / b^{2}+x_{3}^{2} / c^{2}<1$, $a \geq b \geq c$ for the general ellipsoid $E$. It follows easily from Theorem 1 in $\S 3$ above that (i) if $a \rightarrow \infty$ with $b$ and $c$ fixed then $K_{I}(E)$ and $K_{o}(E)$ are bounded, while the methods of proof in Theorem 3 show that (ii) if $a \rightarrow \infty$ and $b \rightarrow \infty$ with $c$ fixed then both coefficients become infinite. These observations were also made by Gehring in a recent treatment [7], his proof of (ii) being based on Theorem 3 of [19] and Theorem 2.3 of [10].

Michigan State University

East Lansing, Michigan, USA 


\section{References}

[1] Ahlfors, L. V., On quasiconformal mappings - J. d'Analyse Math. 3 (1954), 1 - 58.

[2] Anderson, G. D., Symmetrization and extremal rings in space (to appear).

[3] -》- Inequalities for elliptic functions - Amer Math. Monthly 74 (1967), $1072-$ 1074.

[4] Bowman, F., Introduction to Elliptic Functions with Applications - Dover, New York, 1961.

[5] Byrd, P. F. and M. D. Friedman, Handbook of Elliptic Integrals for Engineers and Physicists - Springer, Berlin, 1954.

[6] Fuglede, B., Extremal length and functional completion - Acta Math. 98 (1957), $171-219$.

[7] Gehring, F. W., Coefficients of quasiconformality of domains in three space Contemporary Problems in Theory Anal. Functions (Internat. Conf., Erevan, 1965), pp. 83-88. Izdat. "Nauka", Moscow, 1966.

[8] -》- Extremal length definitions for the conformal capacity of rings in space Mich. Math. J. 9 (1962), 137-150.

[9] -»- Rings and quasiconformal mappings in space - Trans. Amer. Math. Soc. 103 (1962), 353-393.

[10] -»- and J. VÄISÄLÄ, The coefficients of quasiconformality of domains in space Acta Math. 114 (1965), 1-70.

[11] Greenhill, A. G., The Applications of Elliptic Functions - Dover, New York, 1959.

[12] GRötzsch, H., Über möglichst konforme Abbildungen von schlichten Bereichen Ber. Verh. sächs. Akad. Wiss. Leipzig, Math.-phys. Kl. 84 (1932), 114-120.

[13] Kober, H., Dictionary of Conformal Representations - Dover, New York, 1957.

[14] Nehari, Z., Conformal Mapping - McGraw-Hill, New York, 1952.

[15] Nevandinna, R., Eindeutige Analytische Funktionen - Springer, Berlin, 1953.

[16] Saks, S., Theory of the Integral - Hafner, New York, 1937.

[17] Teichmüller, O., Extremale quasikonforme Abbildungen und quadratische Differentiale - Preuss. Acad. Math.-naturw. Kl. 22 (1940), 1-197.

[18] V̈̈rs̈̈LÄ, J., On quasiconformal mappings in space, Ann. Acad. Sci. Fenn. Ser. AI, 298 (1961), 1-36.

[19] - - On quasiconformal mappings of a ball - Ann. Acad. Sci. Fenn. Ser. AI, 304 (1961), $1-7$. 